\title{
An energy estimate for the complex Monge-Ampère operator
}

\author{
by Urban Cegrell and Leif Persson (Umeå)
}

\begin{abstract}
We prove an energy estimate for the complex Monge-Ampère operator, and a comparison theorem for the corresponding capacity and energy. The results are pluricomplex counterparts to results in classical potential theory.
\end{abstract}

Introduction. Recall that in classical potential theory, a positive measure $\mu$ is said to have finite energy if

$$
\int-G_{\Omega}(x, y) d \mu(x) d \mu(y)<\infty,
$$

where $G_{\Omega}$ is the Green function for the domain $\Omega$. It is shown that

$$
\int-G_{\Omega}(x, y) d \mu(x) d \nu(y)
$$

defines an inner product on the linear space of measures spanned by the measures of finite energy. In particular, we have the Cauchy-Schwarz inequality

$$
\left(\int-G_{\Omega} d \mu d \nu\right) \leq\left(\int-G_{\Omega} d \mu d \mu\right)^{1 / 2}\left(\int-G_{\Omega} d \nu d \nu\right)^{1 / 2}
$$

In this paper, we prove the following analogue of this inequality for the complex Monge-Ampère operator:

TheOREM 1.1. Let $\Omega$ be a domain in $\mathbb{C}^{n}, n \geq 2$. Suppose $u, v \in \mathrm{PSH} \cap$ $L^{\infty}(\Omega)$ with $\lim _{z \rightarrow \xi} u(z)=\lim _{z \rightarrow \xi} v(z)=0, \forall \xi \in \partial \Omega$. If $p \geq 1,0 \leq j \leq n$, then

$$
\begin{aligned}
& \int(-u)^{p}\left(d d^{c} u\right)^{j} \wedge\left(d d^{c} v\right)^{n-j} \\
& \quad \leq D_{p, j}\left(\int(-u)^{p}\left(d d^{c} u\right)^{n}\right)^{(p+j) /(n+p)}\left(\int(-v)^{p}\left(d d^{c} v\right)^{n}\right)^{(n-j) /(n+p)}
\end{aligned}
$$

1991 Mathematics Subject Classification: Primary 32F07; Secondary 31C10.

Key words and phrases: capacity, complex Monge-Ampère operator, energy estimate, plurisubharmonic function. 
where $D_{p, j}=p^{(p+j)(n-j) /(p-1)}$ for $p>1$ and $D_{p, j}=\exp (1+j)(n-j)$ for $p=1$.

For the classical notation of energy and Green potentials we refer to Landkof [6], and for the pluripotential theory to the survey article by Bedford $[1]$.

2. Proof of the theorem. In order to be able to integrate by parts, we first assume that

$$
\int_{\Omega}\left(\left(d d^{c} u\right)^{n}+\left(d d^{c} v\right)^{n}\right)<\infty .
$$

Then for the mixed terms we have

$$
\int_{\Omega}\left(d d^{c} u\right)^{j} \wedge\left(d d^{c} v\right)^{n-j} \leq \int_{\Omega}\left(d d^{c}(u+v)\right)^{n}<\infty, \quad 0 \leq j \leq n,
$$

where the last inequality is obtained from the comparison principle and the assumption above (cf. [5]). For let $\mu=\left(d d^{c}(u+v)\right)^{n}$ and choose $1<\alpha<2$ such that $\mu\{u=\alpha v\}=0$. Then $\mu \Omega=\mu\{(1+\alpha) u / \alpha<u+v\}+\mu\{(1+\alpha) v<$ $u+v\}$, and thus $\mu \Omega \leq 3^{n} \int_{\Omega}\left(\left(d d^{c} u\right)^{n}+\left(d d^{c} v\right)^{n}\right)$ by the comparison principle, which proves the boundedness of the mixed terms.

Since $d^{c} u \wedge\left(d d^{c} u\right)^{j-1} \wedge\left(d d^{c} v\right)^{n-j}$ is a positive measure on $\{u=-\varepsilon\}$ (cf. [4]), we have

$$
\begin{aligned}
0 & \leq \int_{\{u=-\varepsilon\}}(-v)^{p} d^{c} u \wedge\left(d d^{c} u\right)^{j-1} \wedge\left(d d^{c} v\right)^{n-j} \\
& \leq \sup \left\{(-v(z))^{p} \mid u(z)=-\varepsilon\right\} \cdot \int_{\Omega}\left(d d^{c} u\right)^{j} \wedge\left(d d^{c} v\right)^{n-j} \rightarrow 0, \quad \varepsilon \searrow 0 .
\end{aligned}
$$

Therefore, we can integrate by parts in this case. Define

$$
\begin{aligned}
& x_{j}=\log \int(-u)^{p}\left(d d^{c} u\right)^{j} \wedge\left(d d^{c} v\right)^{n-j}, \\
& y_{j}=\log \int(-v)^{p}\left(d d^{c} v\right)^{j} \wedge\left(d d^{c} u\right)^{n-j} .
\end{aligned}
$$

Then integration by parts and Hölder's inequality give

$$
\begin{aligned}
\int(-u)^{p}\left(d d^{c} u\right)^{j} \wedge & \left(d d^{c} v\right)^{n-j} \\
= & -\int d v \wedge d^{c}(-u)^{p} \wedge\left(d d^{c} u\right)^{j} \wedge\left(d d^{c} v\right)^{n-j-1} \\
= & \int v d d^{c}(-u)^{p} \wedge\left(d d^{c} u\right)^{j} \wedge\left(d d^{c} v\right)^{n-j-1} \\
= & p(p-1) \int v(-u)^{p-2} d u \wedge d^{c} u \wedge\left(d d^{c} u\right)^{j} \wedge\left(d d^{c} v\right)^{n-j-1} \\
& +p \int(-v)(-u)^{p-1}\left(d d^{c} u\right)^{j+1} \wedge\left(d d^{c} v\right)^{n-j-1} \\
& \leq p \int(-v)(-u)^{p-1}\left(d d^{c} u\right)^{j+1} \wedge\left(d d^{c} v\right)^{n-j-1}
\end{aligned}
$$




$$
\begin{aligned}
& \leq\left(p \int(-v)^{p}\left(d d^{c} u\right)^{j+1} \wedge\left(d d^{c} v\right)^{n-j-1}\right)^{1 / p} \\
& \times\left(p \int(-u)^{p}\left(d d^{c} u\right)^{j+1} \wedge\left(d d^{c} v\right)^{n-j-1}\right)^{(p-1) / p} .
\end{aligned}
$$

Taking logarithms, we get

$$
x_{j} \leq \frac{p-1}{p} x_{j+1}+\frac{1}{p} y_{n-j-1}+\log p
$$

and

$$
y_{j} \leq \frac{p-1}{p} y_{j+1}+\frac{1}{p} x_{n-j-1}+\log p .
$$

In matrix notation,

$$
S\left(\begin{array}{c}
x_{0} \\
y_{0} \\
\vdots \\
x_{n} \\
y_{n}
\end{array}\right) \leq \log p\left(\begin{array}{c}
1 \\
\vdots \\
1
\end{array}\right)
$$

where $S$ is the $2 n \times(2 n+2)$ matrix

$$
S=\left(\begin{array}{ccccccccccc}
1 & 0 & \frac{1-p}{p} & 0 & 0 & \cdots & 0 & 0 & -\frac{1}{p} & 0 & 0 \\
0 & 1 & 0 & \frac{1-p}{p} & 0 & \cdots & 0 & -\frac{1}{p} & 0 & 0 & 0 \\
0 & 0 & 1 & 0 & \frac{1-p}{p} & \cdots & -\frac{1}{p} & 0 & 0 & 0 & 0 \\
\vdots & \vdots & \vdots & \vdots & \vdots & \ddots & \vdots & \vdots & \vdots & \vdots & \vdots \\
\vdots & \vdots & \vdots & \vdots & \vdots & \ddots & \vdots & \vdots & \vdots & \vdots & \vdots \\
\vdots & \vdots & \vdots & \vdots & \vdots & \ddots & \vdots & \vdots & \vdots & \vdots & \vdots \\
0 & 0 & -\frac{1}{p} & 0 & 0 & \cdots & 1 & 0 & \frac{1-p}{p} & 0 & 0 \\
0 & -\frac{1}{p} & 0 & 0 & 0 & \cdots & 0 & 1 & 0 & \frac{1-p}{p} & 0 \\
-\frac{1}{p} & 0 & 0 & 0 & 0 & \cdots & 0 & 0 & 1 & 0 & \frac{1-p}{p}
\end{array}\right)
$$

Let $A$ denote the left $2 n \times 2 n$ submatrix of $S$. We will find that $A$ is invertible and that $A^{-1}$ has nonnegative elements. So multiplication of the system (2.2) with $A^{-1}$ will preserve the inequality and give a reduced row-echelon form. To this end consider the system of equations

$$
A\left(\begin{array}{c}
x_{0} \\
y_{0} \\
\vdots \\
x_{n-1} \\
y_{n-1}
\end{array}\right)=\left(\begin{array}{c}
c_{0} \\
d_{0} \\
\vdots \\
c_{n-1} \\
d_{n-1}
\end{array}\right) .
$$


A calculation shows that then

$$
\begin{aligned}
x_{j}= & \frac{n-j}{(p-1)(p+n)} \sum_{k=0}^{j-1}(k+1) c_{k} \\
& +\frac{p+j}{(p-1)(p+n)} \sum_{k=j}^{n-1}(p-1+n-k) c_{k} \\
& +\frac{n-j}{(p-1)(p+n)} \sum_{k=n-j}^{n-1}(p-1+n-k) d_{k} \\
& +\frac{p+j}{(p-1)(p+n)} \sum_{k=0}^{n-j-1}(k+1) d_{k},
\end{aligned}
$$

and similarly for $y_{j}$. This shows that $A^{-1}$ exists and has nonnegative elements. It follows from (2.3) that

$$
A^{-1} S=\left(\begin{array}{ccccccc}
I & 0 & 0 & \cdots & 0 & 0 & A_{0} \\
0 & I & 0 & \cdots & 0 & 0 & A_{1} \\
\vdots & \vdots & \vdots & \ddots & \vdots & \vdots & \vdots \\
0 & 0 & 0 & \cdots & I & 0 & A_{n-2} \\
0 & 0 & 0 & \cdots & 0 & I & A_{n-1}
\end{array}\right)
$$

where $I$ is the $2 \times 2$ identity matrix and

$$
A_{j}=-\left(\begin{array}{ll}
\frac{p+j}{p+n} & \frac{n-j}{p+n} \\
\frac{n-j}{p+n} & \frac{p+j}{p+n}
\end{array}\right) .
$$

Then (2.2) implies that

$$
A^{-1} S\left(\begin{array}{c}
x_{0} \\
y_{0} \\
\vdots \\
x_{n} \\
y_{n}
\end{array}\right) \leq \log p A^{-1}\left(\begin{array}{c}
1 \\
\vdots \\
1
\end{array}\right)
$$

To compute the right hand side of (2.5), we have to find

$$
A^{-1}\left(\begin{array}{c}
1 \\
\vdots \\
1
\end{array}\right)=\left(\begin{array}{c}
x_{0}^{\prime} \\
y_{0}^{\prime} \\
\vdots \\
x_{n-1}^{\prime} \\
y_{n-1}^{\prime}
\end{array}\right) \text {. }
$$


Thus we put $c_{k}=d_{k}=1$ in $(2.3)$ and get

$$
x_{j}^{\prime}=y_{j}^{\prime}=\frac{(p+j)(n-j)}{p-1} .
$$

We substitute (2.7) and (2.6) in (2.5) and obtain

$$
\begin{aligned}
& x_{j}-\frac{p+j}{p+n} x_{n}-\frac{n-j}{p+n} y_{n} \leq \frac{(p+j)(n-j)}{p-1} \log p, \\
& y_{j}-\frac{n-j}{p+n} x_{n}-\frac{p+j}{p+n} y_{n} \leq \frac{(p+j)(n-j)}{p-1} \log p .
\end{aligned}
$$

This concludes the proof for the case $p>1$ and the extra assumption (2.1). Since the integrals are continuous in $p$, and since

$$
\lim _{p \rightarrow 1} \frac{\log p}{p-1}=1
$$

the inequality also holds for $p=1$. To complete the proof of the theorem, we have to remove the assumption (2.1). We can assume that

$$
\int\left((-u)^{p}\left(d d^{c} u\right)^{n}+(-v)^{p}\left(d d^{c} v\right)^{n}\right)<\infty,
$$

otherwise there is nothing to prove. Let $\varepsilon>0$ be given an let $u_{r}$ denote the usual regularization

$$
u_{r}(z)=\int u(z-r \xi) \phi(\xi) d V(\xi),
$$

where $V$ is the Lebesgue measure on $\mathbb{C}^{n}$, and $\phi$ is a fixed radial, nonnegative, smooth and compactly supported function in the unit ball of $\mathbb{C}^{n}$ with $\int \phi d V=1$. Let $\omega \Subset \Omega$ be a strictly pseudoconvex domain containing $\{u<-\varepsilon / 4\}$. Then $u_{r} \in \operatorname{PSH}(\omega) \cap C^{\infty}(\bar{\omega})$ if $r<d\left(\omega,{ }^{\mathrm{c}} \Omega\right)$, and we define

$$
u_{r, \varepsilon}^{\omega}= \begin{cases}u_{r} & \text { if } u_{r}<-\varepsilon \\ \varepsilon h_{\left\{u_{r}<-\varepsilon\right\}}^{\omega} & \text { if } u_{r} \geq-\varepsilon\end{cases}
$$

where $h_{E}^{\omega}$ is the relative extremal function

$$
h_{E}^{\omega}(z)=\sup \left\{\phi(z)|\phi \in \operatorname{PSH}(\omega), \phi \leq 0, \phi|_{E} \leq-1\right\}
$$

with respect to $\omega$. By Sard's theorem, the boundary of $\left\{u_{r}<-\varepsilon\right\}$ is a smooth manifold for all $\varepsilon$ outside a set of Lebesgue measure zero. We consider only those $\varepsilon$ 's. Then $\lim _{\left\{u_{r} \leq-\varepsilon\right\} \ni \xi \rightarrow z} h_{\left\{u_{r}<-\varepsilon\right\}}^{\omega}(\xi)=-1$ for all $z \in \overline{\left\{u_{r}<-\varepsilon\right\}}$, so $u_{r, \varepsilon}^{\omega}$ is plurisubharmonic on $\omega$. Now,

$$
\begin{aligned}
\int_{\omega}\left(-u_{r, \varepsilon}^{\omega}\right)^{p}\left(d d^{c} u_{r, \varepsilon}^{\omega}\right)^{n} & =\int_{\left\{u_{r}<-\varepsilon\right\}} \ldots+\int_{\left\{u_{r} \geq-\varepsilon\right\}} \ldots \\
& \leq \int_{K}\left(-u_{r}\right)^{p}\left(d d^{c} u_{r}\right)^{n}+\varepsilon^{p} \int_{\left\{u_{r}=-\varepsilon\right\}}\left(d d^{c} u_{r, \varepsilon}^{\omega}\right)^{n}
\end{aligned}
$$


for all compact sets $K$ in $\omega$ containing $\{u<-\varepsilon\}$. Furthermore,

$$
\begin{aligned}
\int_{\omega}\left(d d^{c} u_{r, \varepsilon}^{\omega}\right)^{n} & =\int_{\omega}\left(d d^{c} \varepsilon h_{\left\{u_{r}<-\varepsilon\right\}}^{\omega}\right)^{n}=\int_{\left\{u_{r}=-\varepsilon\right\}}\left(d d^{c} \varepsilon h_{\left\{u_{r}<-\varepsilon\right\}}^{\omega}\right)^{n} \\
& \leq \int_{\left\{u<(\varepsilon / 4) h_{\left\{u_{r}<-\varepsilon\right\}}^{\omega}-\varepsilon / 4\right\}}\left(d d^{c} \varepsilon h_{\left\{u_{r}<-\varepsilon\right\}}^{\omega}\right)^{n} \\
& =4^{n} \int_{\left\{u<(\varepsilon / 4) h_{\left\{u_{r}<-\varepsilon\right\}}^{\omega}-\varepsilon / 4\right\}}\left(d d^{c}\left(\frac{\varepsilon}{4} h_{\left\{u_{r}<-\varepsilon\right\}}^{\omega}-\frac{\varepsilon}{4}\right)\right)^{n} \\
& \leq 4^{n} \int_{\{u<-\varepsilon / 4\}}\left(d d^{c} u\right)^{n}
\end{aligned}
$$

by the comparison principle. Combining these two inequalities, we get

$$
\int_{\omega}\left(-u_{r, \varepsilon}^{\omega}\right)^{p}\left(d d^{c} u_{r, \varepsilon}^{\omega}\right)^{n} \leq \int_{K}\left(-u_{r}\right)^{p}\left(d d^{c} u_{r}\right)^{n}+\varepsilon^{p} \int_{\{u<-\varepsilon / 4\}}\left(d d^{c} u\right)^{n} .
$$

We now let $r \searrow 0$; then $u_{r, \varepsilon}^{\omega}$ decreases to

and

$$
u_{\varepsilon}^{\omega}= \begin{cases}u & \text { if } u<-\varepsilon \\ \varepsilon h_{\{u<-\varepsilon\}}^{\omega} & \text { if } u \geq-\varepsilon\end{cases}
$$

$$
\int_{\omega}\left(-u_{\varepsilon}^{\omega}\right)^{p}\left(d d^{c} u_{\varepsilon}^{\omega}\right)^{n} \leq \int_{K}(-u)^{p}\left(d d^{c} u\right)^{n}+\varepsilon^{p} \int_{\{u<\varepsilon / 4\}}\left(d d^{c} u\right)^{n}
$$

so if we let $\omega$ and $K$ increase to $\Omega$, then $u_{\varepsilon}^{\omega}$ decreases to $u_{\varepsilon}^{\Omega}$ and

$$
\int\left(-u_{\varepsilon}^{\Omega}\right)^{p}\left(d d^{c} u_{\varepsilon}^{\Omega}\right)^{n} \leq \int(-u)^{p}\left(d d^{c} u\right)^{n}+\varepsilon^{p} \int_{\{u<\varepsilon / 4\}}\left(d d^{c} u\right)^{n} .
$$

If we now let $\varepsilon \searrow 0$ then

$$
\lim _{\varepsilon \rightarrow 0} \int\left(-u_{\varepsilon}^{\Omega}\right)^{p}\left(d d^{c} u_{\varepsilon}^{\Omega}\right)^{n} \leq \int(-u)^{p}\left(d d^{c} u\right)^{n}
$$

and similarly for $v$. Also, by semicontinuity we have

$$
\liminf _{\varepsilon \rightarrow 0} \int\left(-u_{\varepsilon}^{\Omega}\right)^{p}\left(d d^{c} u_{\varepsilon}^{\Omega}\right)^{j} \wedge\left(d d^{c} u_{\varepsilon}^{\Omega}\right)^{n-j} \geq \int(-u)^{p}\left(d d^{c} u\right)^{j} \wedge\left(d d^{c} v\right)^{n-j} .
$$

We have already proved the inequalities for $u_{\varepsilon}^{\Omega}$ and $v_{\varepsilon}^{\Omega}$ so the above inequalities complete the proof of the theorem.

R e mark. The theorem can be generalized to more than two functions. Also, it can be proved that $D_{1, j}=1$ (see [7]).

3. An application. Let $\Omega$ be a strictly pseudoconvex set in $\mathbb{C}^{n}, n \geq 2$, and denote by $P$ the class of bounded plurisubharmonic functions $\phi$ on $\Omega$ such that $\lim _{z \rightarrow \xi} \phi(z)=0, \forall \xi \in \partial \Omega$ and $\int_{\Omega}\left(d d^{c} \phi\right)^{n}<\infty$. In analogy with 
the notation of capacity and energy in classical potential theory, we consider the pluricomplex capacity, defined by Bedford and Taylor in [2],

$$
d(F)=\sup \left\{\int_{F}\left(d d^{c} u\right)^{n} \mid u \in P,-1 \leq u \leq 0\right\},
$$

and the pluricomplex energy,

$$
I(F)=\inf \left\{\int-u\left(d d^{c} u\right)^{n} \mid u \in P, \int_{F}\left(d d^{c} u\right)^{n} \geq 1\right\},
$$

of a compact subset $F$ of $\Omega$. If $\int_{F}\left(d d^{c} u\right)^{n}=0, \forall u \in P$, we say that $F$ has infinite energy; this happens exactly when $F$ is pluripolar.

TheOREm 3.1. Suppose that $F$ is not pluripolar. Then

$$
D_{1,0}^{-(n+1) / n} \leq d(F)^{1 / n} I(F) \leq 1 .
$$

Proof. Let $\psi=h_{F}^{*} / d(F)^{1 / n} \in P$, where $h_{F}^{*}$ denotes the smallest upper semicontinuous majorant of the relative extremal function $h_{F}=h_{F}^{\Omega}$ defined by (2.9). Then $\operatorname{supp}\left(d d^{c} \psi\right)^{n} \subset F$ and $\int_{F}\left(d d^{c} \psi\right)^{n}=1$ by [2]. Therefore,

$$
I(F) \leq \frac{1}{d(E)} \int-\frac{h_{F}^{*}}{d(F)^{1 / n}}\left(d d^{c} h_{F}^{*}\right)^{n}=\frac{1}{d(F)^{1 / n}}
$$

since $h_{F}^{*}=-1$ on $F$ outside a pluripolar set. This proves the last inequality in $(3.1)$.

To prove the first inequality we use Theorem 1.1. If $u \in P$ with $\int_{F}\left(d d^{c} u\right)^{n} \geq 1$, then

$$
\begin{aligned}
1 \leq \int-h_{F}\left(d d^{c} u\right)^{n} & \leq D_{1,0}\left(\int-h_{F}\left(d d^{c} h_{F}\right)^{n}\right)^{1 /(n+1)}\left(\int-u\left(d d^{c} u\right)^{n}\right)^{n /(n+1)} \\
& =D_{1,0} d(F)^{1 /(n+1)}\left(\int-u\left(d d^{c} u\right)^{n}\right)^{n /(n+1)}
\end{aligned}
$$

so

$$
D_{1,0}^{-(n+1) / n} \leq d(F)^{1 / n} \int-u\left(d d^{c} u\right)^{n} .
$$

Taking infimum with respect to $u$ we get the first inequality in (3.1), and the proof of the theorem is complete.

Remark. By [7], $D_{1,0}=1$, so we have in fact

$$
d(F)^{1 / n} I(F)=1 \text {. }
$$

This is the pluricomplex counterpart of the classical fact that capacity times energy equals 1 (cf. [3], p. 20). For further results on pluricomplex energy, see $[5]$. 


\section{References}

[1] E. Bedford, Survey of pluri-potential theory, in: Several Complex Variables, Proc. Mittag-Leffler Institute, 1987-88, J. E. Fornaess (ed.), Math. Notes 38, Princeton Univ. Press, 1993, 48-97.

[2] E. Bedford and B. A. Taylor, A new capacity for plurisubharmonic functions, Acta Math. 149 (1982), 1-40.

[3] L. Carleson, Selected Problems on Exceptional Sets, Van Nostrand, Princeton, N.J., 1967.

[4] U. Cegrell, The symmetric pluricomplex Green function, in: Banach Center Publ. 31, Inst. Math., Polish Acad. Sci., Warszawa, 1995, 135-141.

[5] -, Pluricomplex energy, Acta Math., to appear.

6] N. S. Landkof, Foundations of Modern Potential Theory, Springer, 1972.

[7] L. Persson, A Dirichlet principle for the complex Monge-Ampère operator, Research Report No. 8, 1997, Dept. Math., Umeå University.

Department of Mathematics

Umeå University

S-901 87 Umeå, Sweden

E-mail: Urban.Cegrell@mathdept.umu.se

leifp@abel.math.umu.se

Reçu par la Rédaction le 10.10.1996 